\title{
Emergency department non-contrast computed tomography for suspicion of obstructive urolithiasis: Yield and consequences
}

Ziv Savin; ${ }^{1}$ Snir Dekalo; ${ }^{1}$ Eran Schreter $;{ }^{1}$ Reuben Ben-David $;{ }^{1}$ Ismail Masarwa; ${ }^{1}$ Adva CahenPeretz; ${ }^{1}$ Sharon A. Greenberg $;{ }^{2}$ Galit Aviram $;{ }^{3}$ Ofer Yossepowitch $;{ }^{1}$ Mario Sofer ${ }^{1,4}$

${ }^{1}$ Department of Urology, Tel-Aviv Sourasky Medical Center, Tel Aviv, Israel; ${ }^{2}$ Department of Emergency Medicine, Tel-Aviv Sourasky Medical Center, Tel Aviv, Israel; ${ }^{3}$ Department of Radiology, Tel-Aviv Sourasky Medical Center, Tel Aviv, Israel; ${ }^{4}$ Department of Endourology Unit, Tel-Aviv Sourasky Medical Center, Tel Aviv, Israel

Cite as: Savin Z, Dekalo S, Schreter e, et al. Emergency department non-contrast computed tomography for suspicion of obstructive urolithiasis: Yield and consequences. Can Urol Assoc J 2022 February 28; Epub ahead of print. http://dx.doi.org/10.5489/cuaj.7570

Published online February 28, 2022

Corresponding author: Dr. Ziv Savin, Department of Urology, Tel Aviv Sourasky Medical Center, Tel Aviv, Israel; zivsavin23@gmail.com

$* * *$

\section{Abstract}

Introduction: We aimed to analyze patterns of referral, yield, and clinical implications of noncontrast computed tomography (NCCT) in the acute evaluation of flank pain suspected as obstructive urolithiasis (OU) in a high-volume emergency department (ED).

Methods: The study comprised 506 consecutive NCCTs performed in the ED over four months. Detection rates of OU, incidental, and alternative findings were calculated. Imaging signs suspicious for recent passage of stones were considered positive for OU, while renal stones without signs of obstruction were considered unrelated to the acute presentation. OU, other findings requiring hospitalization, and incidental findings warranting further workup were considered situations in which NCCTs were warranted.

Results: NCCTs confirmed an OU diagnosis in $162(32 \%)$ patients and non-clinically significant nephrolithiasis in 125 (25\%). They revealed other findings in 108 (21\%) patients, including 42 (8\%) with clinically significant incidental findings and $26(5 \%)$ with alternative diagnoses requiring hospitalization. NCCTs were entirely negative in $111(22 \%)$ patients. Corroboration of these outcomes, together with overlapping of OU, incidental, and alternative significant findings in some patients resulted in an overall justified NCCT request rate of $44 \%$. 
Conclusions: The yield of NCCT performed in acute presentations of flank pain suspected as OU is relatively low, and over one-half of the scans are unwarranted. The pattern of requesting NCCT in the ED needs refinement to avoid abuse that may lead to radiation overexposure, psychological burden, physical harm, and financial overload.

\section{Introduction}

Non-contrast computed tomography (NCCT) is considered the standard of reference imaging study for the diagnosis of urolithiasis. ${ }^{1-4}$ Its high sensitivity and specificity, broad spectrum of indications, ease of processing, and rapid interpretation led to wide hospital dotation, availability, and use. ${ }^{2}$ Consequently, NCCT utilization for the detection of obstructive urolithiasis (OU) in emergency departments (ED) has increased considerably, and it has become the most preferred initial diagnostic imaging modality in many institutions. ${ }^{5-7}$ NCCT provides invaluable information that may dictate the approach to the treatment of urolithiasis when used with caution and high clinical expertise for the assessment of acute flank pain. In addition, it may detect alternative causes for flank pain. When performed at a low level of suspicion and/or weak clinical basis, however, they may detect incidental findings that may lead to unjustified medical evaluations. ${ }^{8-10}$ We aimed to analyze the pattern of requests and yield of NCCTs in diagnosing OU in the acute setting, and estimated its appropriateness and the associated clinical implications.

\section{Methods}

This retrospective study was approved by the medical center's Institutional Review Board and patients or the public were not involved in the design, or conduct, or reporting, or dissemination plans of our research. We identified all NCCTs performed in the ED between January $1^{\text {st }}$ and April $30^{\text {th }}, 2018$. The ED in our institution is staffed by physicians certified in emergency medicine who perform the triage and initial assessment and establish the working diagnosis, followed by other medical specialty consultations if needed. All NCCTs requested for flank pain suspected as being due to renal colic were included in the study.

All the included patients were scanned by a multi-detector CT scanner (Brilliance; Philips Medical Systems, Cleveland, OH, USA) with 64 detector rows. The reconstructed slice thickness was 2.0 with an increment of $1.0 \mathrm{~mm}$.The NCCT protocol consisted of scanning from the lung bases to the groins, in one breath hold. The tube voltage was set at $120 \mathrm{Kv}$ while the tube current was $200 \mathrm{mAs}$ with automatic exposure adjustment according to patient size. The standard examination reports included the CT dose index (CTDI; mGy), dose-length product (DLP; $\mathrm{mGy} / \mathrm{cm}$ ) and effective dose (EDose; $\mathrm{mSv}$ ) which was calculated multiplying DLP by the standard conversion coefficient for a CT abdomen/pelvis $(\kappa=0.015)$. No oral or intravenous 
contrast was used, and the patient was in a prone position to facilitate the distinction between a stone located at the ureterovesical junction and one located in the bladder.

Ureteral stones and/or signs suspected as recent passage of stones (e.g.,

uretero/hydronephrosis, perinephric edema, periureteral edema and stone in the bladder) were considered as validating the diagnosis of OU. ${ }^{11}$ Renal stones without signs of obstruction were considered unrelated to the acute pain. Accordingly, the reported NCCT findings were classified as confirmatory for $\mathrm{OU}$, incidental, alternative, or negative. Incidental findings were categorized by significance according to the American College of Radiology white papers ${ }^{12}$. Alternative findings were defined as the ones that could explain the patient's symptoms in the absence of OU.

The performance of an NCCT scan was considered as having been justified when the findings included $\mathrm{OU}$, alternative findings requiring hospitalization, and clinically significant incidentalomas warranting further evaluation. The estimation of detection rates of NCCTs for OU, categorization of additional findings, and analysis of the clinical yield and warranting of NCCT were carried out by means of SPSS software version 25 (IBM Corp., Armonk, NY, USA, 2017).

\section{Results}

The study comprised 506 consecutive NCCTs of patients presenting to the ED with flank pain of suspected OU origin. The mean age of the study population was 50 years $(\mathrm{SD}=18)$ with a predominance for male sex (62\% males; $38 \%$ females). The average EDose per NCCT was $8 \mathrm{mSv}$ (range 5.3-11.2). OU was identified in $162 / 506$ patients (32\%) and non-obstructive nephrolithiasis was detected in 125/506 (25\%). NCCTs revealed 167 additional finding with no urolithiasis in 108/506 patients (21\%), and they were negative in 111/506 (22\%) patients. There were 76 patients with incidental findings (15\%) and 65 patients (13\%) with alternative findings among those 108 patients (Tables 1 and 2). Non-significant incidental findings included adrenal adenomas, hernia, and uterine fibroids, while significant incidentalomas were primarily lesions suspected for malignancy, such as renal and hepatic masses, pulmonary nodules, and lymphadenopathy. Forty-six significant incidental findings that required further ambulatory evaluation (e.g., imaging, biopsy, or specialist consultation) were found in 42/506 patients (8\%, Table 1). Alternative pathologies requiring hospitalization were detected in $26 / 506$ patients (5\%, Table 2), with biliary disease being the most common among them. Performance of NCCTs in the acute setting was warranted in 225/506 cases (44\%), with 162 (32\%) due to OU, $42(8 \%)$ due to significant incidentalomas, and $26(5 \%)$ due to alternative causes needing hospitalization. There were 5 cases with more than 1 finding, yielding an overlapping rate of $1 \%$. Among the 281/506 unwarranted cases (56\%), 111 (22\%) had negative scans, $125(25 \%)$ had nonobstructive nephrolithiasis, 34 (7\%) had non-significant incidental findings and $39(8 \%)$ had an alternative diagnosis allowing discharge, with overlapping rate of $6 \%$ (30 cases). 


\section{Discussion}

A CT scan provides a wide range of diagnostic capabilities at a high level of cost effectiveness. As such, it has become the principal imaging tool in many institutions. Its availability and diagnostic advantages led to an exponential rise of its use in the ED setting in which a simple scanning procedure may rapidly and effectively deliver essential information for an accurate diagnosis. ${ }^{12-14}$ The NCCT scan was largely adopted by ED staffs due to its proven superiority over ultrasonographic and conventional kidney-ureter-bladder studies in clarifying the cause of acute flank pain. ${ }^{3-4}$

However, it appears that this success led to a progressive decrease in selectivity for requesting the exam, resulting in greater numbers of scans that were extraneous. ${ }^{7,13}$ The monthly average number of NCCTs performed in the ED for flank pain has increased from 15-20 to 80150 studies in a single decade. ${ }^{8,9,16-18}$ Hyams et al. demonstrated that NCCT utility rates increased in flank pain patients from $20 \%$ in 2000 to $45 \%$ in $2008 .{ }^{14}$ In opposition, other studies that focused on OU suggested a decreasing rate of correlation between clinical suspicion and imaging confirmation. The detection rate of OU in patients presenting with flank pain was 55$72 \%$ in the late 1990 's and it decreased to $37-48 \%$ from the beginning of the millennium..$^{2-4,8,15,16}$ Our OU detection rate of only $32 \%$ in a high-volume emergency setting with a mean of 127 NCCTs per month may reflect this global decreasing rate. It may be also influenced by the business of our urban facility covering a population of half million people, with persistent over agglomeration. In such condition, a quick one breath-hold efficient imaging may resolve almost instantaneously a case of flank pain maintaining an adequate flow of patients and reducing patient's time spend in ED. We originally aimed to investigate the practice of NCCT referral in our ED with an eye towards revealing some abuse of the imaging services. However, this turned out to be beyond the scope of our study. We believe that refined instructions of the ED staff to enhance its competence both in ordering NCCTs and in the potential risks related to NCCTs are probably a key to improving referral practices, but further studies are needed to address these issues.

The detection rate for incidentalomas was $15 \%$, however, only $8 \%$ were of clinical significance that indicated further evaluation. Incidental findings are well known by-products of abdominopelvic CT scans, ${ }^{12}$ and ongoing improvement in CT resolution has led to a marked increase in the detection of findings unrelated to the primary objective. ${ }^{17,18}$ Incidental findings may assist in diagnosing a significant disease earlier in its course. On the other hand, it may reportedly lead to a cascade of testing, including invasive procedures, that promotes financial costs up to US $\$ 50,000$ per patient and may cause harm to patients both physically and psychologically. ${ }^{24,19}$ In addition, it exposes patients to unnecessary radiation and wastes time and effort on the part of physicians. ${ }^{19,20}$ Importantly, our results suggest that only few patients who do not have urolithiasis would benefit from undergoing an NCCT scan in the context of incidentalomas. 
In the current study, NCCTs diagnosed alternative findings that were followed by hospitalization in $5 \%$ of the patients, among them appendicitis, biliary disease, and diverticulitis. Given its cross-sectional nature, NCCTs have the ability to suggest an alternative diagnosis, since a variety of diseases can mimic renal colic. Radiologists must be familiar with the spectrum of differential diagnoses that may be detected with this modality in order to accurately identify the source of flank pain. ${ }^{13,21} \mathrm{We}$ had assumed that an alternative diagnosis requiring hospitalization would be considered as being one that justified the NCCT scan despite the absence of urolithiasis. However, the 26 (5\%) patients found on NCCTs to have highly significant morbidities needed extension to intravenous contrast administration protocols in order to arrive at the proper diagnosis.

Many earlier reports highlighted the presence of various additional findings in NCCTs performed for acute flank pain in the ED. They categorized the findings by systems and significance without providing any guidelines for the optimal indications or algorithm for requesting them. ${ }^{8-10,15,16,21-24}$ In our study, we justified the performance of NCCTs according to 3 criteria: the presence of $\mathrm{OU}$, incidental findings requiring a workup, and alternative findings requiring hospitalization. The referral to NCCT imaging studies emerged as being justified in $44 \%$ of our series. We consider that this rate is actually too low, given that most incidental findings proved to be benign, and that most of the alternative pathologies should have been scanned with intravenous contrast from the outset.

Several studies have attempted to increase the detection rate of significant findings on NCCTs by corroborating their request with clinical features, such as hematuria, nausea, personal and family history, and laboratory results. Validation of these scoring tools, however, resulted in unsatisfactory prediction characteristics. ${ }^{25-29}$ In addition, it is possible that lack of a urologic consultation before deciding upon referral to NCCT contributed to the apparently low detection rate and overuse. Nevertheless, NCCT in the ED usually puts an end to the diagnosis for patients who are apprehensive about their condition. Given the delays of outpatient healthcare systems, the rapidity and $100 \%$ sensitivity and specificity of NCCT in the diagnosis of urinary stones justifies its use when appropriately selected. Considering that ED is a high turnover acute setting of presentation, diagnosis and referral for treatment, our algorithm does not include standardized low dose NCCT protocols. Although these protocols may reduce the effective dose radiation to $0.48 \mathrm{mSv}$ per NCCT, they are associated with a significant decrease in their yield. ${ }^{30}$ A study assessing the diagnostic effectiveness of low dose NCCT for renal colic/flank pain in 33 patients revealed a reduction of sensitivity, specificity, positive predictive value and negative predictive value for stone detection to $72 \%, 94 \%, 93 \%$ and $75 \%$, respectively. In addition, 35\% of extraurinary findings, including acute appendicitis, adrenal adenomas, ovarian dermoid and gallstones were missed by the low radiation NCCT. ${ }^{31}$ Thus, in our opinion these protocols should be use with caution in ED, however they may have an important role for follow-up of patients with known active stone events or for assessing stone-free status after treatments of urinary stones. Intriguingly, despite the fact that low-dose NCCT protocols were initially described more than a 
decade ago, they are quoted today at $\angle 4 \mathrm{mSv}$ and their implementation rates do not exceed $8 \% .{ }^{32,33}$ Further strategies and alternative clinical algorithms are needed to reduce NCCT use without increasing the risk of significant misdiagnosis in the ED.

Our study is limited by its having been carried out in a single-center and its retrospective design, as well as by the inability to objectively analyze the clinical assessment that led to the suspicion of OU and request for an NCCT scan. In addition, the NCCTs were interpreted by different radiologists, thus potentially exposing the findings to some inter- and intra-observer variability bias, as reported in the literature. ${ }^{25}$ Lastly, for the methodology of our study we decided to validate $\mathrm{OU}$ in the presence of signs for recent passage of stone and to consider non-obstructing renal stones not related to the acute pain. However, uretero/hydronephrosis, perinephric edema and periureteral edema may be rather signs of pyelonephritis ${ }^{34}$ with no OU, and rarely non-obstructing renal stone does cause some pain. This fact might have changed slightly our justification rates. Despite these limitations, we believe that the consecutive layout and the high-volume ED of our large tertiary referral academic center represent real-life practice and mitigate some of these limitations.

\section{Conclusions}

The detection rates for OU on NCCTs are decreasing in parallel with its increasing rate of utility in the ED. It appears that more than half of those NCCTs are not warranted, thereby generating non-significant findings and leading to radiology overloading, unnecessary radiation exposure, unjustified expenditures, psychological burden, and physical harm. Reducing their overuse yet minimalizing misdiagnosis should be a priority while developing novel clinical algorithms for identifying the precise source of acute flank pain. 


\section{References}

1. Smith RC, Rosenfield AT, Choe KA, et al. Acute flank pain: comparison of noncontrast-enhanced CT and intravenous urography. Radiology. Published online 1995. doi:10.1148/radiology.194.3.7862980

2. Fielding JR, Steele G, Fox LA, Heller H, Loughlin KR. Spiral computerized tomography in the evaluation of acute flank pain: A replacement for excretory urography. J Urol. Published online 1997. doi:10.1016/S0022-5347(01)64676-7

3. Yilmaz S, Sindel T, Arslan G, et al. Renal colic: Comparison of spiral CT, US and IVU in the detection of ureteral calculi. Eur Radiol. Published online 1998. doi:10.1007/s003300050364

4. Niall O, Russell J, Macgregor R, Duncan H, Mullins J. A comparison of noncontrast computerized tomography with excretory urography in the assessment of acute flank pain. J Urol. Published online 1999. doi:10.1016/S0022-5347(01)61942-6

5. Brenner DJ, Hall EJ. Computed Tomography - An Increasing Source of Radiation Exposure. N Engl J Med. 2007;357(22):2277-2284. doi:10.1056/NEJMra072149

6. Schoenfeld EM, Pekow PS, Shieh M-S, Scales CD, Lagu T, Lindenauer PK. The Diagnosis and Management of Patients with Renal Colic across a Sample of US Hospitals: High CT Utilization Despite Low Rates of Admission and Inpatient Urologic Intervention. Remuzzi G, ed. PLoS One. 2017;12(1):e0169160. doi:10.1371/journal.pone.0169160

7. Ziemba JB, Sterling ME, Mucksavage P. Care of acute renal colic: A survey of emergency medicine physicians. Can J Urol. Published online 2016.

8. Hoppe H, Studer R, Kessler TM, Vock P, Studer UE, Thoeny HC. Alternate or Additional Findings to Stone Disease on Unenhanced Computerized Tomography for Acute Flank Pain Can Impact Management. J Urol. Published online 2006. doi:10.1016/S0022-5347(05)00987-0

9. Ahmad NA, Ather MH, Rees J. Incidental diagnosis of diseases on un-enhanced helical computed tomography performed for ureteric colic. BMC Urol. Published online 2003. doi:10.1186/1471-2490-3-2

10. Ather MH, Memon W, Rees J. Clinical impact of incidental diagnosis of disease on non-contrast-enhanced helical CT for acute ureteral colic. Semin Ultrasound, CT MRI. Published online 2005. doi:10.1053/j.sult.2004.10.005

11. AA S. Renal colic: pathophysiology, diagnosis and treatment. Eur Urol. 2001;39(3):241-249. doi:10.1159/000052446

12. Berland LL, Silverman SG, Gore RM, et al. Managing incidental findings on abdominal CT: White paper of the ACR incidental findings committee. J Am Coll Radiol.

Published online 2010. doi:10.1016/j.jacr.2010.06.013

13. Reddy S. State of the art trends in imaging renal of colic. Emerg Radiol. 2008;15(4):217-225. doi:10.1007/s10140-008-0705-6

14. Schoenfeld EM, Pekow PS, Shieh M-S, Scales CD, Lagu T, Lindenauer PK. The Diagnosis and Management of Patients with Renal Colic across a Sample of US Hospitals: High CT Utilization Despite Low Rates of Admission and Inpatient Urologic Intervention. Remuzzi G, ed. PLoS One. 2017;12(1):e0169160. 
doi:10.1371/journal.pone. 0169160

15. Agarwal MD, Levenson RB, Siewert B, Camacho MA, Raptopoulos V. Limited added utility of performing follow-up contrast-enhanced CT in patients undergoing initial nonenhanced CT for evaluation of flank pain in the emergency department. Emerg Radiol. 2015;22(2):109-115. doi:10.1007/s10140-014-1259-4

16. Katz DS, Scheer M, Lumerman JH, Mellinger BC, Stillman CA, Lane MJ. Alternative or additional diagnoses on unenhanced helical computed tomography for suspected renal colic: Experience with 1000 consecutive examinations. Urology. Published online 2000. doi:10.1016/S0090-4295(00)00584-7

17. Yee J, Kumar NN, Godara S, et al. Extracolonic Abnormalities Discovered Incidentally at CT Colonography in a Male Population. Radiology. 2005;236(2):519-526. doi:10.1148/radiol.2362040166

18. Pickhardt PJ, Hanson ME, Vanness DJ, et al. Unsuspected Extracolonic Findings at Screening CT Colonography: Clinical and Economic Impact ${ }^{1}$. Radiology. 2008;249(1):151-159. doi:10.1148/radiol.2491072148

19. Ganguli I, Simpkin AL, Lupo C, et al. Cascades of Care After Incidental Findings in a US National Survey of Physicians. JAMA Netw open. 2019;2(10):e1913325. doi:10.1001/jamanetworkopen.2019.13325

20. Siddiki H, Fletcher JG, McFarland B, et al. Incidental findings in CT colonography: literature review and survey of current research practice. J Law Med Ethics. 2008;36(2):320-331, 213. doi:10.1111/j.1748-720X.2008.00276.x

21. Rucker CM, Menias CO, Bhalla S. Mimics of renal colic: alternative diagnoses at unenhanced helical CT. Radiographics. 2004;24 Suppl 1(suppl_1):S11-28; discussion S28-33. doi:10.1148/rg.24si045505

22. Eshed I, Kornecki A, Rabin A, Elias S, Katz R. Unenhanced spiral CT for the assessment of renal colic. How does limiting the referral base affect the discovery of additional findings not related to urinary tract calculi? Eur J Radiol. Published online 2002. doi:10.1016/S0720-048X(01)00404-1

23. Cullen IM, Cafferty F, Oon SF, et al. Evaluation of Suspected Renal Colic with Noncontrast CT in the Emergency Department: A Single Institution Study. J Endourol. 2008;22(11):2441-2446. doi:10.1089/end.2008.0120

24. Samim M, Goss S, Luty S, Weinreb J, Moore C. Incidental Findings on CT for Suspected Renal Colic in Emergency Department Patients: Prevalence and Types in 5,383 Consecutive Examinations. J Am Coll Radiol. 2015;12(1):63-69. doi:10.1016/j.jacr.2014.07.026

25. Daniels B, Gross CP, Molinaro A, et al. STONE PLUS: Evaluation of Emergency Department Patients with Suspected Renal Colic, Using a Clinical Prediction Tool Combined with Point-of-Care Limited Ultrasonography. Ann Emerg Med. Published online 2016. doi:10.1016/j.annemergmed.2015.10.020

26. Rule AD, Lieske JC, Li X, Melton LJ, Krambeck AE, Bergstralh EJ. The ROKS nomogram for predicting a second symptomatic stone episode. J Am Soc Nephrol. Published online 2014. doi:10.1681/ASN.2013091011

27. Hernandez N, Song Y, Noble VE, Eisner BH. Predicting ureteral stones in emergency department patients with flank pain: an external validation of the STONE score. World $J$ 
Urol. 2016;34(10):1443-1446. doi:10.1007/s00345-016-1760-3

28. Moore CL, Bomann S, Daniels B, et al. Derivation and validation of a clinical prediction rule for uncomplicated ureteral stone--the STONE score: retrospective and prospective observational cohort studies. BMJ. 2014;348:g2191. doi:10.1136/bmj.g2191

29. The evaluation and management of urolithiasis in the ED: A review of the literatureClinicalKey. Accessed April 14, 2020.

https://vpn.tasmc.org.il/,DanaInfo=www.clinicalkey.com,SSL+\#!/content/playContent/1 -s2.0-S0735675718300032?returnurl=null\&referrer=null

30. S J, OJ O, MM M, MF M. Strategies for dose reduction with specific clinical indications during computed tomography. Radiogr (London, Engl 1995). 2020;26 Suppl 2:S62-S68. doi:10.1016/J.RADI.2020.06.012

31. PD M, KP M, SA H, et al. Non-contrast CT at comparable dose to an abdominal radiograph in patients with acute renal colic; impact of iterative reconstruction on image quality and diagnostic performance. Insights Imaging. 2014;5(2):217-230. doi:10.1007/S13244-014-0310-Z

32. DT T, S Z, M U, et al. Computed Tomography Radiation Exposure Among Referred Kidney Stone Patients: Results from the Registry for Stones of the Kidney and Ureter. $J$ Endourol. 2019;33(7):619-624. doi:10.1089/END.2019.0091

33. K W, P K, M S, et al. Evaluation of Kidney Stones with Reduced-Radiation Dose CT: Progress from 2011-2012 to 2015-2016-Not There Yet. Radiology. 2018;286(2):581589. doi:10.1148/RADIOL.2017170285

34. T Y, T T, R F, et al. Usefulness of computed tomography in the diagnosis of acute pyelonephritis in older patients suspected of infection with unknown focus. Acta Radiol. Published online 2021. doi:10.1177/0284185120988817 
Figures and Tables

\begin{tabular}{|c|c|c|}
\hline Type of incidental finding & Overall & Led to further workup \\
\hline \multicolumn{3}{|l|}{ Genitourinary, $\mathrm{n}$} \\
\hline Complex renal cyst & 1 & 1 \\
\hline Renal mass & 4 & 4 \\
\hline Angiomyolipoma & 1 & 0 \\
\hline Adnexal cyst & 1 & 0 \\
\hline Uterine fibroids & 5 & 0 \\
\hline Other & 6 & 0 \\
\hline \multicolumn{3}{|l|}{ Gastrointestinal, $n$} \\
\hline Cholelithiasis & 2 & 0 \\
\hline Hepatic lesion & 10 & 6 \\
\hline Pancreatic mass / cyst & 2 & 2 \\
\hline Pancreatic ductal dilatation & 1 & 1 \\
\hline Atrophic pancreas & 1 & $\mathbf{1}$ \\
\hline Esophageal dilatation & 1 & 1 \\
\hline Bowel intussusception & 1 & 1 \\
\hline Appendix mucocele & 1 & 1 \\
\hline Other & 5 & 0 \\
\hline \multicolumn{3}{|l|}{ Pulmonary, $n$} \\
\hline Nodule & 12 & 12 \\
\hline Pleural effusion & 3 & 0 \\
\hline Metastasis & 1 & $\mathbf{1}$ \\
\hline Other & 3 & 0 \\
\hline \multicolumn{3}{|l|}{ Adrenal, $\mathrm{n}$} \\
\hline Hematoma & 1 & 1 \\
\hline Mass & 1 & 1 \\
\hline Adenoma/hyperplasia & 9 & 0 \\
\hline \multicolumn{3}{|l|}{ Other, $\mathrm{n}$} \\
\hline Lymphadenopathy & 8 & 8 \\
\hline Splenic lesion & 2 & 2 \\
\hline Aortic aneurysm & 2 & 2 \\
\hline Retroperitoneal sarcoma & 1 & 1 \\
\hline Hernia (umbilical, inguinal) & 13 & 0 \\
\hline Fat necrosis & 1 & 0 \\
\hline Bone findings & 3 & 0 \\
\hline Total findings & 102 & 46 \\
\hline Total patients & 76 & 42 \\
\hline
\end{tabular}




\begin{tabular}{|c|c|c|}
\hline Alternative findings & Overall & Hospitalizations \\
\hline Urinary tract infection & 4 & 1 \\
\hline SOL of bladder & 1 & 1 \\
\hline Adnexal lesion & 5 & $\mathbf{0}$ \\
\hline Hepatitis & 1 & 1 \\
\hline Biliary disease & 8 & 4 \\
\hline Pancreatitis & 1 & 1 \\
\hline Appendicitis & 3 & 3 \\
\hline Diverticulitis & 7 & 3 \\
\hline Colitis/enteritis & 3 & 1 \\
\hline GI perforation & 2 & 2 \\
\hline Epiploic appendagitis & 4 & $\mathbf{0}$ \\
\hline Intestinal foreign body & 1 & 1 \\
\hline Sclerosing mesenteritis & 1 & $\mathbf{0}$ \\
\hline Pneumonia & 3 & $\mathbf{1}$ \\
\hline Pleural effusion & 2 & $\mathbf{0}$ \\
\hline Pulmonary embolism & 1 & 1 \\
\hline Bone metastasis & 3 & 2 \\
\hline Sacroiliitis & 2 & $\mathbf{0}$ \\
\hline Vertebral fracture & 2 & 1 \\
\hline Spinal stenosis & 1 & $\mathbf{0}$ \\
\hline Discopathy & 5 & $\mathbf{0}$ \\
\hline Vasculitis & 1 & 1 \\
\hline Inguinal hernia & 3 & 1 \\
\hline Splenic infract & 1 & 1 \\
\hline Total & 65 & 26 \\
\hline
\end{tabular}

GI: gastrointestinal; SOL: space-occupying lesion. 